\title{
Adenosine Modulates the Oocyte Developmental Competence by Exposing Stages and Synthetic Blocking during In Vitro Maturation
}

\author{
'Yong-Pil Cheon \\ Division of Development and Physiology, Department of Biology, Institute for Basic Sciences, \\ Sungshin Women's University, Seoul 136-742, Republic of Korea
}

\begin{abstract}
Purine metabolism is known factor for nuclear maturation of oocytes through both follicle cells and oocyte itself. However, it is largely unknown the roles of purine metabolism in the oocyte competence for fertilization and early development. In this study, the effects of adenosine in oocyte competence for development were examined using adenosine and its synthetic inhibitors. Adenosine treatment from GV intact stage for $18 \mathrm{hr}$ (fGV) caused of decrease the fertilization rate but of increase the cleavage rate compared from the other stage treatment groups. Hadacidin did not effect on fertilization rate but increased cleavage rate without stage specificity. Adenosine did not block the effects of hadacidin with the exception of fGV group. By the inhibition of purine synthetic pathways the fertilization rate was decreased in the fGV and fGVB groups but increased in the fMII group. Exogenous adenosine caused of decrease fertilization rate in the fGVB group but increase in the fMII group. Cleavage rate was dramatically increased in the adenosine treatment with synthetic inhibitors. It means that the metabolism of purine has stage specific effects on fertilization and cleavage. Exogenous adenosine had only can improve oocyte developmental competence when it treated at GV intact stage. On the other hand, endogenous synthesis in all maturation stage caused of increase the cleavage rate without effects on fertilization. These data suggest that adenosine at GV stage as a paracrine fashion and inhibitions of endogenous adenosine in all stage improve oocyte developmental competence.. Key words : Adenosine, De novo synthesis, Oocyte, Developmental competence
\end{abstract}

\section{INTRODUCTION}

It is evaluated that the developmental competence of oocyte has been improved by modulation of cyclic adenosine monophosphate (cAMP) levels during in vitro maturation (Gilchrist, 2011; Ezoe et al., 2015). Addition of a cAMP modulator forskolin or 3-isobutyl-1-methyl-xanthine (IBMX) to the maturation media improves the developmental competence of vitrified-thawed GV oocytes as in natural GV oocytes (Ezoe et al., 2015). It based on the LH or FSH works through cAMP mediated signaling pathways (Dekel \& Kraicer, 1978; Sela-Abramovich et al., 2006; Conti et al., 2012) and maturation promoting factor (Ezoe et al., 2015).

In meiotic arrest, spontaneous maturation is observed after releasing from follicle (Edwards, 1962; Pincus \& Enzmann, 1935). So, follicular environment is important for oocyte competence. From 1970s, it is suggested that maintenance of appropriate cAMP levels is critical factor for meiotic resumption regulation (Luciano et al., 2011;

\footnotetext{
Manuscript received May 28, 2016, Received in revised form June 05, 2016, Accepted June 12, 2016

† Corresponding Author : Yong Pil Cheon, Division of Development and Physiology, Department of Biology, Institute for Basic Sciences, Sungshin Women's University, Seoul 136-742, Republic of Korea. Tel. : +82-2-920-7639, Fax : +82-2-920-2093, E-mail: ypcheon@sungshin.ac.kr

This is an Open Access article distributed under the terms of the Creative Commons Attribution Non-Commercial License (http:// creativecommons.org/licenses/by-nc/3.0) which permits unrestricted non-commercial use, distribution, and reproduction in any medium, provided the original work is properly cited.
} 
Richard 2007; Cho et al., 1974). cAMP levels are under the multiple factors such as $\mathrm{G}$ protein-coupled receptor 3 (GPR3), GRP8, cyclases, phosphodiesterases, and growth factors such as EGF-like peptides (Richard, 2007; Conti et al., 2005; Kawamura et al., 2004). Adenosine increases FSH-stimulated accumulation of cAMP in cumulus-oocytecomplexs (COC) and markedly inhibits oocyte maturation in a dose-dependent manner (Miller \& Behrman, 1986).

On the other hand, de novo synthesis of purines in somatic compartment of follicle and the timing and duration of such synthesis determines whether meiotic resumption will be suppressed or promoted in hormone mediated actions (Downs \& Verhoeven, 2003; Downs, 2000). The purine synthesis by de novo pathway is important in maintenance of meiotic arrest under the hormone. Purine de novo synthesis is nearly doubled in OCC by FSH treatment, and this response was completely prevented by adenosine (Downs, 2000). FSH had no effect on hypoxanthine salvage, although adenosine reduced this activity by $98 \%$ (Downs, 2000). hCG-induced maturation is blocked by adenosine as effectively as by the purine de novo synthesis inhibitor, azaserine (Downs, 2000). Cheon et al. (1997) revealed that the azaserine, de novo synthetic inhibitor, at GV intact stage increases the nuclear maturation and polar body extrusion rates. On the other hand, it inhibited the polar body extrusion rate if it is treated from GVB stage. So it is suggested that purine metabolism may concern with the maturation process with other signaling mediators. Salvage synthesis of purine is so far confused in oocyte maturation, although it is used to recover bases and nucleosides and can be converted back into nucleotides. Downs suggested that salvage pathway may be not required for meiotic induction $(1997 \mathrm{a}, \mathrm{b})$ but he could not conformed (Downs, 2000).

The possible roles of purine metabolism including adenosine on germinal vesicle breakdown is mediated the cAMP. cAMP levels also showed fluctuation during maturation (Webb et al., 2008; Hegele-Hartung et al., 1999). On the other hand, it has been suggested a role as signaling mediator in oocyte maturation. However, it is largely un-known whether exogenous adenosine works as messenger during oocyte maturation or after maturation and its roles in oocyte developmental competence. In here, the possible roles of adenosine as messenger and its metabolism were evaluated with combination of purine synthesis metabolic inhibitors and adenosine.

\section{MATERIALS AND METHODS}

\section{Animals}

All animals used in this study were CD-1 mice (6-8 weeks old) and were handled according to the National Institutes of Health Standards for the use and care of animals. The animal protocols were approved by Sunshin University Institutional Animal Care and Use Committee. Animals were maintained on a 14L:10D light dark cycle under standard conditions. Food and water were provided ad libitium. For IVF sperm was obtained from same strain male mice between 8 and 12 weeks old.

\section{Oocyte collection and in vitro maturation (IVM)}

Immature CD-1 mice (21-day-old) were injected with 5 IU of pregnant mare serum gonadotropin (Sigma) to stimulate the follicular development at 1:00 pm. Animals were sacrifice $46 \mathrm{hr}$ later and oocytes were got through puncture the antral follicles with fine needle. The cumuli were removed physically using capillary pipet. Denuded germinal vesicle intact oocytes were pooled and washed three times in fresh medium and used. These processes were per-formed within 10 minutes. Oocytes were randomly allotted to treatment groups of about 100 per group. Groups were distinguished like followings: from $\mathrm{GV}$ intact group (fGV), from GVB group (fGVB), and from MII group (fMII). In fGV, chemicals were treated from GV intact stage and cultured for $18 \mathrm{hr}$. In GVB, chemicals were treated from GVB stage for $9 \mathrm{hr}$. In fMII, chemicals were treated 
in MII oocytes for $2 \mathrm{hr}$ (from $15 \mathrm{hr}$ after maturation induction). After culture, the MII stage oocytes were washed in fresh medium at least 5 times. Groups of 10 washed denuded oocytes were matured in $10 \mu \mathrm{L}$ drops of equilibrated BWW medium containing $0.4 \%$ BAS covered with mineral oil at $37^{\circ} \mathrm{C}, 5 \% \mathrm{CO}_{2}$ in air. To evaluate the possible roles of adenosine and its synthetic inhibitors, $750 \mu \mathrm{M}$ adenosine (A), $20 \mu \mathrm{M}$ azacerine (Aza), $20 \mu \mathrm{M}$ hadacidine (Had), and $10 \mu \mathrm{M}$ mycophenolic acid (MA) were used.

\section{IVF and embryo culture}

The effect of adenosine and its synthetic inhibitors on oocyte developmental competence was assessed by examining the capacity of the oocyte to support preimplantation embryo development following in vitro maturation. All the media used were BWW medium containing 0.4\% BSA. Data presented percentage of 10 replicate experiments. About 100 oocytes were used per treatment group. IVF was performed as described in the Manipulating the Mouse Embryo (Hogan et al., 1994). Fertilization was carried out for $4 \mathrm{hr}$ and then the eggs were washed with medium to remove sperms. 10 zygotes were place in $10 \mu \mathrm{L}$ drops of equilibrated BWW medium containing $0.4 \%$ BAS covered with mineral oil at $37^{\circ} \mathrm{C}, 5 \% \mathrm{CO}_{2}$ in air. Fertilization was assessed by the presence of second polar body and the development of 2-cell stage embryos the next day.

\section{Statistical analysis}

Data were represented as percentage. Data on fertilization and cleavage rate were analyzed using Chi-square test. $P<$ 0.05 was assumed to indicate statistical significance.

\section{RESULTS}

\section{Exogenous adenosine has no effect after GVB on}

Most of the immature oocytes which were cultured with $750 \mu \mathrm{M}$ adenosine were failed to become MII stage oocytes
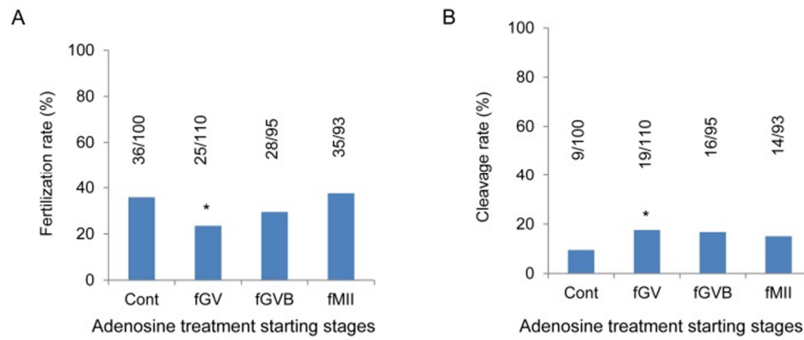

Fig. 1. Effects of adenosine treated during maturation for 18, 9, or $2 \mathrm{hr}$ on fertilization (A) and cleavage (B). fGV is means that GV intact oocytes were cultured in the medium containing $750 \mu \mathrm{M}$ adenosine for $18 \mathrm{hr}$. fGVB is means that GVB oocytes were cultured in the medium containing $750 \mu \mathrm{M}$ adenosine for $9 \mathrm{hr}$. fMII is means that MII oocytes were treated $2 \mathrm{hr}$ with $750 \mu \mathrm{M}$ adenosine. Cont, control; $\mathrm{fGV}$, from germinal vesicle stage; fGVB, from germinal vesicle breakdown stage; fMII, from metaphase II stage. ${ }^{*}: P<0.05$ control vs experimental groups $\left(\chi^{2}\right.$-test)

but after GVB stage adenosine did not showed specific effects as mentioned in previous report (Cheon et al., 1997). In the fGV groups, adenosine was treated from GV intact stage for $18 \mathrm{hr}$ to induce spontaneous maturation. In the MII stage oocytes getting at $18 \mathrm{hr}$ after incubation (fGV) the fertilization was significantly low compared with other groups. However, the cleavage rate was significantly high compared with the other groups (Fig. 1A, B). Adenosine did not increase or decrease the fertilization rate or cleavage rate in the other groups (Fig. 1A, B). It showed that the effects of adenosine on oocyte competence are existing at GV stage.

2. Adenosine de novo inhibition enhancing the cleavage rate

To suppress the endogenous adenosine in oocyte, hadacidin was employed. Hadacidin can suppress synthesis of adenosine from inosine monophosphate (IMP). Hadacidin did not increase or decrease the fertilization rates by the treatment starting stages (Fig. 2A). However, the cleavage rate was 

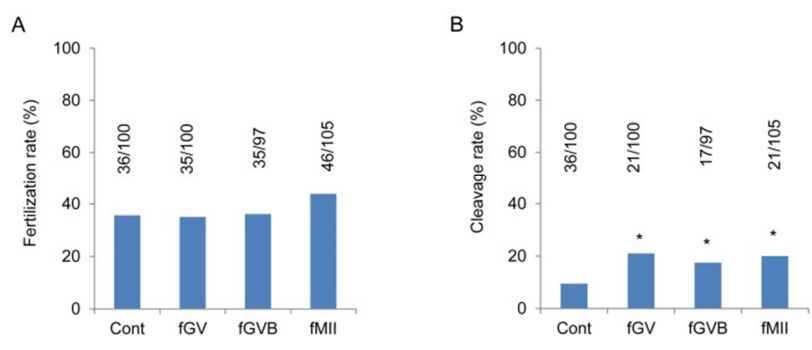

Fig. 2. Effects of hadacidin on fertilization (A) and cleavage (B) which were treated during maturation for 18,9 , or $2 \mathbf{h r}$. fGV is means that GV intact oocytes were cultured in the medium containing 20 $\mu \mathrm{M}$ hadacidin for $18 \mathrm{hr}$. fGVB is means that GVB oocytes were cultured in the medium containing 20 $\mu \mathrm{M}$ hadacidin for $9 \mathrm{hr}$. fMII is means that MII oocytes were treated $2 \mathrm{hr}$ with $20 \mu \mathrm{M}$ hadacidin. MII stage oocytes were involved in experiments. Cont, control; fGV, from germinal vesicle stage; fGVB, from germinal vesicle breakdown stage; fMII, from metaphase II stage. ${ }^{*}: P<0.05$ control vs experimental groups $\left(\chi^{2}\right.$-test).

significantly higher in all three groups, fGV, fGVB and fMII compared with control (Fig. 2B). It clearly showed the inhibition of adenosine synthesis has positive effects on the improve oocyte quality.

\section{Exogenous adenosine did not overcome with the} concentration compensation manners of the hadacidin effects

To know whether exogenous adenosine can block the effects of hadacidin on the oocyte developmental competence, hadacidin and adenosine were co-treated as mentioned at Materials and Methods. Adenosine did not block the effects of hadacidin in fGVB and fMII groups. However, it blocked the effects of hadacidin at fGV. It means that adenosine does not work as quantity compensation manners at $\mathrm{GV}$ stage at least.

\section{Purine synthesis had maturation stage specific} effects on oocyte developmental competence

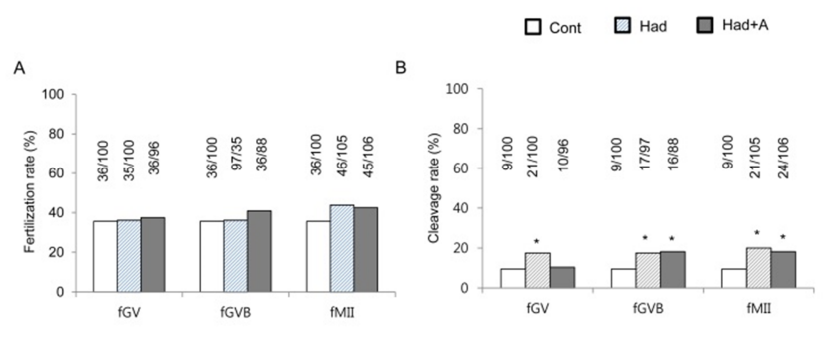

Fig. 3. Combined effects of hadacidin and adenosine on fertilization (A) and cleavage (B) which were treated during maturation for 18,9 , or $2 \mathrm{hr}$. fGV is means that GV intact oocytes were cultured in the medium containing $20 \mu \mathrm{M}$ hadacidin and $750 \mu \mathrm{M}$ adenosine for $18 \mathrm{hr}$. fGVB is means that GVB oocytes were cultured in the medium containing $20 \mu \mathrm{M}$ hadacidin for $9 \mathrm{hr}$. fMII is means that MII oocytes were treated $2 \mathrm{hr}$ with $20 \mu \mathrm{M}$ hadacidin and $750 \mu \mathrm{M}$ adenosine. MII stage oocytes were involved in experiments. Cont, control; fGV, from germinal vesicle stage; fGVB, from germinal vesicle breakdown stage; fMII, from metaphase II stage. " $: P<$ 0.05 control vs experimental groups $\left(\chi^{2}\right.$-test).

To evaluate the possible role adenosine in the oocyte maturation, synthetic pathways were blocked with azacerine, hadacidin, and mycophenolic acid at one time with or without adenosine. Fertilization rates were decreased in fGV and fGVB groups by the blocking of synthesis but increased in fMII group. Adenosine had opposite effect for the synthesis blocking at fGVB and fMII groups (Fig. 4A). Fertilization rate were improved by the adenosine compared with control and synthesis blocking groups only in fMII group (Fig. 4B).

\section{DISCUSSION}

Metabolism of purine nucleotides in oocyte or cumulus is suggested as one of the factors for meiotic arresting. Purine de novo or salvage syntheses are involved in regulation mechanism of oocyte maturation. Purine salvage synthesis supplies adenine or guanine from intermediates in the degradative pathway for nucleotides. Salvage pathways are 


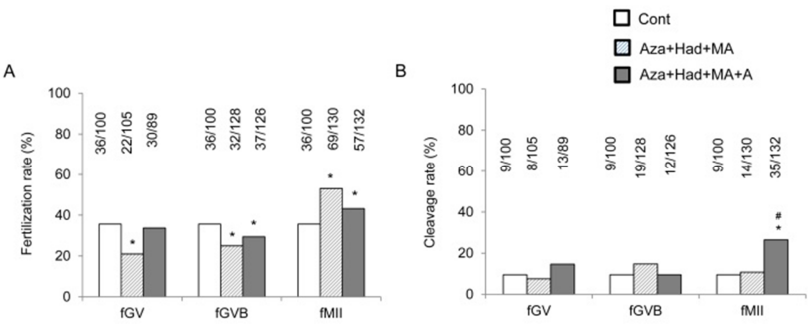

Fig. 4. Combined effects of azacerine, hadacidin, mycophenolic acid, and adenosine on fertilization (A) and cleavage (B) which were treated during maturation for 18,9 , or $2 \mathbf{h r}$. $\mathrm{fGV}$ is means that $\mathrm{GV}$ intact oocytes were cultured for $18 \mathrm{hr}$. fGVB is means that GVB oocytes were cultured for $9 \mathrm{hr}$, fMII is means that MII oocytes were treated $2 \mathrm{hr}$ with $20 \mu \mathrm{M}$ azacerine, $20 \mu \mathrm{M}$ hadacidin, mycophenolic acid, and $750 \mu \mathrm{M}$ adenosine. MII stage oocytes were involved in experiments. Cont, control; fGV, from germinal vesicle stage; fGVB, from germinal vesicle breakdown stage; fMII, from metaphase; Aza, azacerine; Had, hadacidin; MA, mycophenolic acid; A, adenosine. II stage. ${ }^{*}: P<0.05$ control vs experimental groups $\left(\chi^{2}\right.$-test $)$.

used to recover bases and nucleosides. De novo synthesis begins with simple molecules such as amino acids and bicarbonate (Berg et al., 2002). Salvage synthesis is much less costly than the de novo synthesis. Adenylosuccinate synthetase catalyzes the first committed step in the biosynthesis of AMP form IMP. Adenine phosphoribosyl transferase catalyzes the formation of adenylate, whereas hypoxanthine-guanine phosphoribosyl transferase catalyzes the formation of inosinate and guanylate. It has been evaluated that these purine metabolism is important in meiotic arrest.

Previous data showed that adenosine treatment from GV intact stage significantly disturbed the maturation processes after GVB (Cheon et al., 1997). Interestingly, however, in the MII oocytes which were matured in the media containing adenosine, the cleavage rate was significantly higher than the other groups. Exogenous adenosine did not inhibit or accelerate of fertilization rate and cleavage rate of MII stage oocytes which were got from fGVB and fMII eggs. It can be supported by the previously evaluated results; meiotic suppression by cAMP-upregulation agents improves subsequent oocyte developmental competence (Luciano et al., 2011; Nogueira et al., 2003a, b). It means that adenosine work mediator of meiotic arrest as well as oocyte competence.

It has been suggested that adenosine blocks meiotic induction via its pronounced inhibition of the purine and pentose phosphate metabolic pathways (Downs, 2000). On the other hand, it also has been suggested that salvage synthesis of purine is not required for meiotic induction (Downs, 1997a, b). Interestingly, present study showed that the blocking of endogenous adenosine synthesis causes of increase of the cleavage rate. Besides, cotreatment of hadacidin and adenosine did not support quantitative compensation. Those suggest that exogenous adenosine work as messenger not as metabolic substance. It also can be supported by Fig. 4 .

Previously we suggested that adenosine receptors in oocyte may regulate the maturation (Hwang \& Cheon, 2013). It is supported by the present study. In GV intact oocytes adenosine improved fertilization rates without inhibit the effects of hadacidin. It suggests that the adenosine mediate signaling is involved in cytoplasmic maturation and improve developmental competence.

On summary, adenosine improved the cleavage rate when it treated from GV intact stage but not form GVB or MII. By the blocking the endogenous adenosine synthesis, the fertilization rates were increased in all groups, fGV, fGVB, and fMII. Besides, adenosine did not compensation the hadacidine in fGVG and fMII group except in fGV group. Those suggest that adenosine work with paracrine fashion during GV intact stage for oocyte development competence. Blocking de novo synthesis had the treatment stage specific effects on fertilization rate and adenosine only improved the cleavage rate. Put together those results suggest that 
adenosine works with paracrine fashion and adenosine metabolism has stage specific effects on oocyte developmental competence, although further studies in the molecular level are needed.

\section{ACKNOWLDEGEMENT}

This work was supported by a grant from the Sungshin University (2016-1-11-047). None of the authors are involved in any potential financial conflicts of interest.

\section{REFERENCES}

Berg JM, Tymoczko JL, Stryer L (2002) Biochemistry. 5th ed. WH Freeman, New York.

Cheon YP, Kim CH, Mik JE. Kim MK (1997) Effects of adenosine, guanosine nad azaserine on maturation of mouse oocytes in vitro. Korean J Animal Reprod 21: $123-130$.

Cho WK, Stern S, Biggers JD (1974) Inhibition effect of dibutyryl cAMP on mouse oocyte maturation in vitro. $\mathrm{J}$ Exp Zool 187:383-386.

Conti M, Hsieh M, Park JY, Su YQ (2005) Role of the EGF network in ovarian follicles. Mol Endocrinol 20: $715-723$

Conti M, Hsieh M, Zamah AM, Oh JS (2012) Novel signaling mechanisms in the ovary during oocyte maturation and ovulation. Mol Cell Endocrinol 356:65-73.

Dekel N, Kraicer PF (1978) Induciton in vitro of mucification of rat cumulus oophorus by gonadotrophins and adenosine 3',5'-monophosphate. Endocrinology 102:17971802.

Downs SM (1997a) Involvement of purine nucleotide synthetic pathways in gonadotropin-induced meiotic maturation in mouse cumulus cell-enclosed oocytes. Mol Reprod Dev 46:155-167.

Downs SM (1997b) Hypoxanthine regulation of oocyte maturation in the mouse: insights using hypoxanthine phosphoribosyltransferase-deficient animals. Biol Reprod 57:54-62.

Downs SM (2000) Adenosine blocks hormone-inuced meiotic maturation by suppressing purine de novo synthesis. Mol Reprod Dev 56:172-179.

Downs SM, Verhoeven A (2003) Glutamine and the maintenance of meiotic arrest in mouse oocytes: Influence off culture medium, glucose and cumulus cells. Mol Reprod Dev 66:90-97.

Edwars RG (1962) Maturation in vitro of mouse, sheep, cow, pig, rhesus monkey and human ovarian oocytes. Nature 208:349-351.

Ezoe K, Yabuuchi A, Tani T, Mori C, Miki T, Takayama Y, Beyhan Z, Kato Y, Okuno T, Kobayashi T, Kato K (2015) Developmental competence of vitrified $\beta$ warmed bovine oocytes at the germinal-vesicle stage is improved by cyclic adenosine monophosphate modulators during in vitro maturation. PLoS One 12:10(5).

Gilchrist RB (2011) Recent insights into oocyt-follicle cell interactions provide opportunities for the development of new approaches to in vitro maturation. Reprod Fertil Dev 23:23-31

Hegele-Hartung C, Kuhnke J, Lessl M, Grondahl C, Ottesen J, Beier HM, Eisner S, Eichenlaub-Ritter U (1999) Nuclear and cytoplasmic maturation of mouse oocytes after treatment with synthetic meiosis-activating sterol in vitro. Biol Repro 61:1362-1372.

Hogan B, Beddington R, Costantini F, Lacy E (1994) Manipulating the Mouse Embtryo: a Laboratory Manual. $2^{\text {nd }}$ ed. Cold Spring Harbor Laboratory Press, New York.

Hwang H, Cheon YP (2013) Adenosine receptors mediated intracellular calcium in cumulus cells involved in the maintenance of first meiotic arrest. Dev Reprod 17: 141-147.

Kawamura K, Kumagai J, sudo S, Chun SY, Pisarska m, Morita H, Toppari J, Fu P, Wade JD, Bathgate RA, 
Hsueh AJ (2004) Paracrine regulation of mammalian oocyte maturation and male germ cell survival. Proc Natl Acad Sci USA 101:7323-7328.

Luciano AM, Franciosi F, Modina SC, Lodde V (2011) Gap junction-mediated communications regulate chromatin remodeling during bovine oocyte growth and differrentiation through cAMP-dependent mechanism9s). Biol Reprod 85:1252-1259.

Miller JGO, Behrman HR (1986) Oocyte maturation is inhibited by adenosine in the presence of folliclestimulating hormone. Biol Reprod 35:833-837.

Nogueira D, Albano C, Adriaenssens T, Cortvindt R, Bourgain C, Devroey P, Smitz J (2003a) Human oocyte reversibly arrested in prophse I by phosphodiesterase type 3 inhibitor in vitro. Biol Reprod 69:1042-1052.

Nogueira D, Cortvrindt R, De Matos DG, Vanhoutte L,
Smitz J (2003b) Effect of phosphodiesterase type 3 inhibitor on developmental competence of immature mouse oocytes in vitro. Biol Reprod 69:2045-2052.

Pincus J, Enzmann EV (1935) The comparative behavior of mammalian eggs in vitro and in vivo. J Exp Med 62: 665-675.

Richard FJ (2007) Regulation of meiotic maturation. J Anim Sci 85:E4-E6.

Sela-Abramovich S, Edry I, Galiani D, Nevo N, Dekel N (2006) Disruption of gap junctional communication within the ovarian follicle induces oocyte maturation. Endocrinology 147:2280-2286.

Webb RJ, Tinworth L, Thomas GMH, Zaccolo M, Carroll J (2008) Developmentally acquired PKA localization in mouse oocytes and embryos. Dev Reprod 317:36-45. 